\title{
Comparing RLS and LMS Adaptive Equalizers for Large Hydrophone Arrays in Underwater Acoustic Communication Channels
}

\author{
Jacob Rudander* ${ }^{\dagger}$, Thor Husøy*, Pål Orten*† and Paul van Walree * *Kongsberg \\ Maritime, Horten Norway (jacob.hiden.rudander@kongsberg.com) ${ }^{\dagger}$ Oslo \\ University, Oslo Norway ${ }^{\ddagger}$ Norwegian Defence Research Establishment, Horten \\ Norway
}

\begin{abstract}
There is an expected growth in underwater acoustic data traffic. To meet the demand of high data throughput, a migration towards the very high frequency band ( $>100 \mathrm{kHz})$ will be necessary. The increment in frequency comes with two problems; high propagation losses and rapid channel fluctuations. One solution addressing both of these obstacles is the use of large hydrophone arrays in combination with multi-channel equalizers. We will in this paper examine and compare tracking properties and convergence rate of the RLS and LMS adaptive algorithm for use in large multichannel equalizers. Using data from a very high frequency $(250 \mathrm{kHz})$ channel we will show that in our channel, for large hydrophone arrays, RLS does not offers a significant advantage over LMS when it comes to tracking channel variations nor convergence rate.
\end{abstract}

\section{INTRODUCTION}

The interest for coherent mid-range high-speed underwater acoustic communications is expected to grow over the next few years. To meet the demand for high data-rates, a migration towards the very high frequency band $(>100 \mathrm{kHz})$ will be necessary in order to make available wider bandwidths.

In addition to the common traits of underwater acoustic channels [1], the very high frequency channel is characterized by rapid channel fluctuations and a high attenuation which significantly reduces the achievable communication range. In order to batter these effects large hydrophone arrays can be used. This work is about the use of large hydrophone arrays at receiver side. 
The higher attenuation can be compensated for through receiver array gain, coherently combining the received signals. A suitable method for combining signals is a multi-channel equalizer, that in addition will compensate for the acoustic channel inferred multi-path propagation and time variability. A commonly used equalizer design for underwater acoustic communication is the decision feedback equalizer [2], [3], [4], [5]. The filter taps are typically updated using the recursive least square (RLS) algorithm, well known for its rapid convergence rate. But the RLS algorithm, is also very processing intensive and has a complexity that increases quadratic with the number of filter taps. This is not necessarily a problem for single hydrophone solutions or when the size of the hydrophone array is small, but will become a significant problem increasing the number of hydrophones inevitable resulting in unachievable processing demands.

To come around those obstacles it has been suggested to divide the hydrophone array into subarrays each processed by an multichannel equalizer [6], or by prebeamforming to reduce the number of channels (beams) into the equalizer [7]. The first method has a drawback considering the weak signals whereas the latter explicitly requires knowledge of the spatial distribution of the pressure field. Further for very large arrays the number of beams may also grow large in order to avoid highly directive beams which may worsen the performance.

A less processing intensive alternative to the RLS algorithm is the least mean squares (LMS) algorithm, whose processing complexity increases linearly with the number of filter taps. The drawback of LMS is the potentially slower convergence rate, sensitive to the eigenvalue spread of the recieved signals correlation matrix. The reduction in convergence rate could be problematic especially for single hydrophone systems, leaving the receiver unable to track the changes in the channel, with loss of performance as a result.

It should be noted that there are other implementations of the RLS algorithm which are less processing demanding; for example lattice and fast transversal filter based implementations of the RLS algorithm. These implementations are still more complex than LMS which also has the advantage of being possible to implement using solely multiplication and accumulators making it more suitable for real time implementation. Since these less processing intensive versions of RLS algorithm has no extra advantages over RLS [8], besides being just less processing intensive, they will not be considered in this paper whose scope is to compare the tracking and convergence performance of RLS and LMS based multichannel equalizers. The emphasis is on large hydrophone arrays, examining how an increasing number of hydrophones in a fixed aperture affects the choice of receiver adaptive algorithm. 


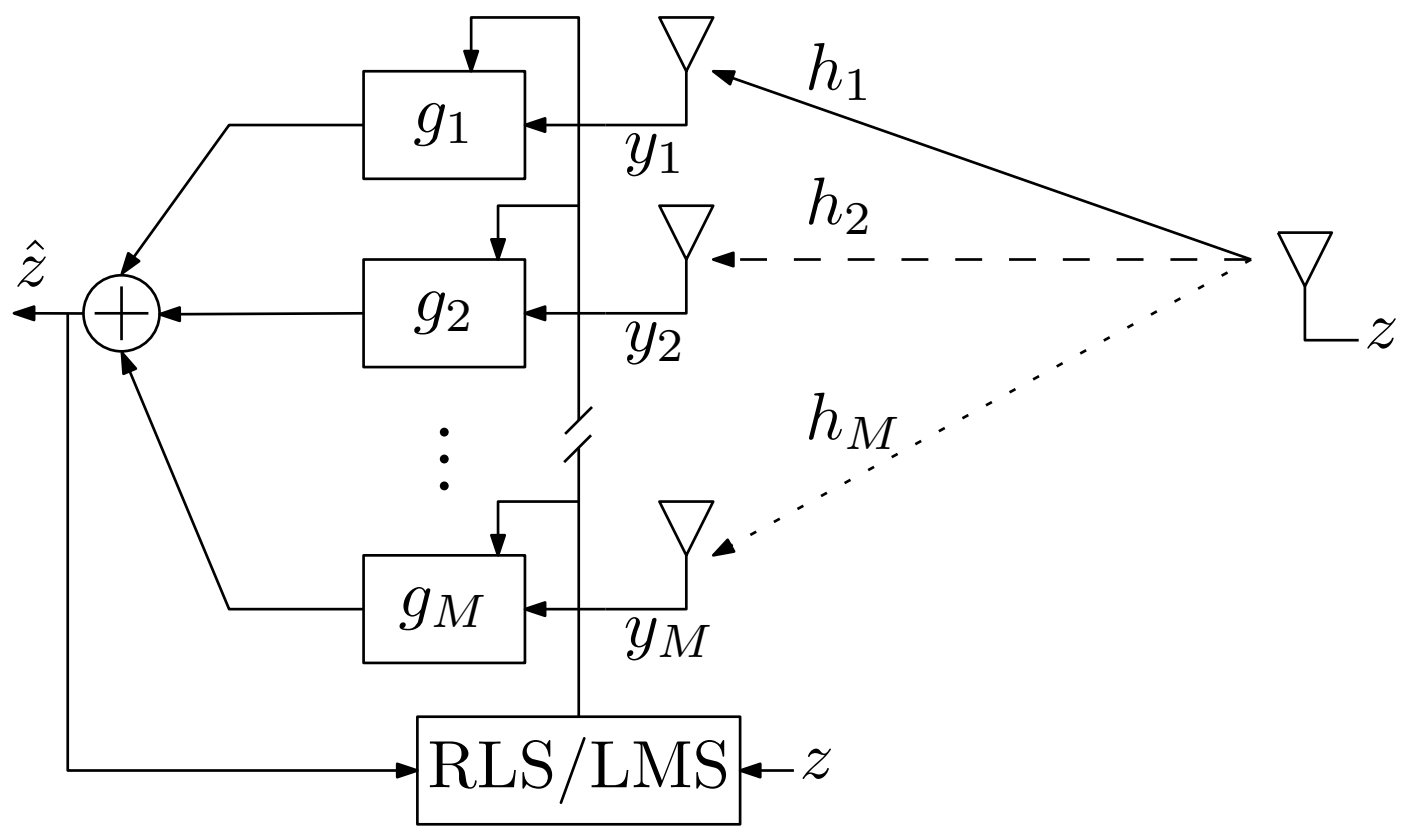

Fig. 1. Multichannel decision feedback equalizer, using either LMS or RLS algorithm for feed-forward filter tap update.

Using a 64 hydrophone line array we will examine, using the same dataset, how the tracking capability and initial convergence rate is effected by the use of the RLS and LMS algorithm. For the comparison we are using real data recorded in slanted very high frequency, $250 \mathrm{kHz}$, rapid fluctuating acoustic channels under various conditions and ranges.

\section{TRACKING RANDOM TIME-VARYING CHANNELS}

In this section a model for the time varying acoustic channel and the used multichannel receiver tracking the channel variations will be described. It is assumed that all signals have been brought to complex baseband, that constant Doppler shifts have been compensated for by means of resampling and that carrier recovery has been properly done. A sketch of the depicted system can be seen in Fig. 1. Let $\mathbf{z}(t)=[z(t+L) \ldots z(t-L+1)]^{\mathrm{T}}$ be a column vector representing the transmitted complex baseband symbols sampled at time instant $t$. $L$ is the length of the acoustic channel in number of samples. The received symbol $y_{m}(t)$ measured at hydrophone $m$ in an $M$ hydrophone array is then a distorted version of $z(t)$, where the distortion is determined by the propagation conditions for the specific acoustic channel. The channel is typically modeled as a time-varying linear filter. Doing so the received symbol at hydrophone 
$m$ can be expressed as

$$
y_{m}(t)=\mathbf{h}_{m}^{\mathrm{H}}(t) \mathbf{z}(t)+n(t)
$$

where, $\mathbf{h}_{m}^{\mathrm{H}}(t)$ is the time-varying acoustic channel impulse response valid at time instant $t$ in vector form of length $L$ and $n(t)$ is the ambient noise.

Typically the channel impulse response will contain several multipath components spread both in time, space and possibly frequency [1], which will significantly distort the symbol.

\section{A. Multichannel equalizer}

In order to recover the transmitted data, the channel impulse response must be compensated for. This can be done by filtering the received symbols with the inverse impulse response $\mathbf{g}_{m}(t)$ of the acoustic channel experienced by hydrophone $m$. The soft symbol estimate, $\hat{z}$, can for a multichannel equalizer be expressed as

$$
\hat{z}(t)=\sum_{m=1}^{M} \mathbf{g}_{m}(t)^{\mathrm{H}} \mathbf{y}_{m}(t),
$$

where $\mathbf{y}_{m}(t)=\left[y_{m}(t)(t+L) \ldots y_{m}(t)(t-L+1)\right]^{\mathrm{T}}$. However, there are two practical problems doing so, 1) the inverse impulse response, $\mathbf{g}_{m}(t)$ is of infinite length and 2) time variability of the channel limit validity of the inverse filter to a finite time.

The solution to the first problem is to truncate the inverse filter constraining its duration to the specified channel length, $L$. The solution to the latter is to adaptive update the inverse filter taps. In this paper we are considering the RLS and LMS algorithm for finding the inverse filter which minimize the minimum mean-square error (MMSE) of the misadjustment $e(t)=z(t)-\hat{z}(t)$. The transmitted symbols $z(t)$ are typically unknown at the receiver and are fed back to the equalizer based on decision made on the soft symbol estimate. However in order to compare solely the performance of the different algorithms, the equalizer will in this work be in training mode, knowing all of the transmitted symbols $z(t)$.

1) LMS algorithm: The main steps of the LMS algorithm can be summarized as

$$
\begin{aligned}
& \hat{z}(t)=\sum_{m=1}^{M} \mathbf{g}_{m}(t)^{\mathrm{H}} \mathbf{y}_{m}(t), \\
& e(t)=z(t)-\hat{z}(t), \\
& \mathbf{g}_{m}(t)=\mathbf{g}_{m}(t-1)+2 \mu \mathbf{y}_{m}(t) e(t),
\end{aligned}
$$

where $\mu$ is the learning rate. Further implementation details of the LMS algorithm can be seen in for example [8]. 
2) RLS algorithm: The main steps of the RLS algorithm can be summarized as

$$
\begin{aligned}
& \hat{z}(t)=\sum_{m=1}^{M} \mathbf{g}_{m}(t)^{\mathrm{H}} \mathbf{y}_{m}(t), \\
& e(t)=z(t)-\hat{z}(t), \\
& \mathbf{R}_{m}^{-1}(t)=\frac{1}{\alpha}\left(\mathbf{R}_{m}^{-1}(t)-\frac{\mathbf{R}_{m}^{-1}(t) \mathbf{y}_{m}(t) \mathbf{y}_{m}^{\mathrm{H}}(t) \mathbf{R}_{m}^{-1}(t)}{\alpha \mathbf{y}_{m}^{\mathrm{H}}(t) \mathbf{R}_{m}^{-1}(t-1) \mathbf{y}_{m}(t)}\right), \\
& \mathbf{g}_{m}(t)=\mathbf{g}_{m}(t-1)+\mathbf{R}_{m}^{-1}(t) \mathbf{y}_{m}(t) e(t) .
\end{aligned}
$$

The forgetting factor $\alpha$ is typically chosen close to unity and $\mathbf{R}_{m}(t)$ represents the estimate of the correlation matrix for the filter input $y_{m}(t)$. Further implementation details can be seen in for example [8].

\section{B. Discussion based on RLS and LMS algorithms}

The most obvious characteristic that distinguish the LMS algorithm from the RLS algorithm is the simplicity of implementation which requires only multiplications and accumulations. However, the simplicity has its price, namely slower convergence compared to RLS and a possibility for being unstable, using inappropriate learning rates.

By using a small value of the learning rate $\mu$ the adaption of the LMS algorithm will be slow. On the other hand assigning a large $\mu$ will increase the convergence rate at the cost of noise amplification. Assigning a too large $\mu$ will cause the algorithm to diverge. In order for the LMS algorithm to be convergent $\mu$ must not exceed the largest eigenvalue of $\mathbf{R}$, the (ensemble averaged) filter input correlation matrix; $\mu<2 \lambda_{\max }$ [8]. Or equivalently $\mu<2 / \sum_{m=1}^{M} P_{m}(t)$ where $P_{m}(t)$ is the instantaneous input power at hydrophone $m$.

The LMS algorithm is therefore not only sensitive to the eigenvalue spread of $\mathbf{R}$. Which causes filter taps corresponding to small eigenvalues to converge at slower rate, leading to long convergence times for channels with a large eigenvalue spread and further making the convergence time channel dependent. But also sensitive to variations in the instantaneous input power, where steep variations of the input signal strength may cause the algorithm to have unpredictable convergence rate, or even worse diverge.

The stability and convergence rate of the RLS algorithm on the other hand is basically insensitive to both variation in the input signal strength and eigenvalue spread. Although the eigenvalue spread affects steady state error, but at a much less extent [8]. The price for this is a higher computational cost, which increases quadratic with the channel length, $L$. 
These advantages makes the RLS algorithm superior of LMS in most situations, although it has been shown that in system identification problems for short varying channels and white input, the RLS and LMS algorithm would have the same tracking performance [9], with a carefully selected learning rate, $\mu$, of the LMS algorithm. The problem is that the underwater acoustic channel is rarely short, nor the input is white. Why RLS often show a higher or equivalent performance compared to LMS in single hydrophone systems and is not an uncommon choice among underwater communication receivers.

For single hydrophone systems and small arrays, RLS would be the natural choice consider its advantages. However, as the number of hydrophones increases the extra computational cost with RLS will eventually be difficult to handle, and LMS should be used. This is not as problematic as it first may seams. First the channel fluctuations will reduce as an effect of spatial averaging where uncorrelated channel fluctuations are averaged over all hydrophones, although being most prominent in short channels, it can reduce the most rapid input power variations. Secondly is the fact that the magnitude of uncorrelated errors among the hydrophones will reduce as $1 / M$ compared to the symbol, and effectively diminish when $M$ grows large. Consequently the meansquare error (MSE) is expected to decrease as $1 / M$. This suggest that even though the difference between RLS and LMS may be large for small hydrophone arrays, the effective difference will be small for large hydrophone arrays, as we will show in this paper.

\section{DATA COLLECTION}

The experiment was conducted during 2016 in the Oslo fjord. The set-up consisted of a single transmitter with a $\alpha=30^{\circ}$ opening angle orientated $\beta=45^{\circ}$ from the seabed, attached to a rigid steel frame placed at the seabed. The receiver with 64 hydrophones arranged in a line array with a spacing ensuring no grating lobes, was mounted on a surface vessel. Fig. 3 depicts the slanted horizontal channel. The transmitter was oriented so that it was pointing in the same direction as the wind, this to allow the surface vessel to slowly drift off with the wind while recording of data was made. The transmitted waveform was binary phase-shift keying (BPSK) modulated using a bit rate of $78.125 \mathrm{kbps}$ and a carrier frequency of $250 \mathrm{kHz}$. For additional details of the experiment the reader is referred to [10].

Shown in Fig. 2 is the power delay profile summarizing the conditions in some of the measured channels. Channels A-C are shallow water channels of increasing range, where the shortest ranges have a direct arrival, followed by a long energy rich tail of reflected arrivals. In channel 
TABLE I

EXPERIMENT CONDITIONS AND PARAMETERS

\begin{tabular}{lcccc}
\hline \hline Channel & $\mathrm{A}$ & $\mathrm{B}$ & $\mathrm{C}$ & $\mathrm{D}$ \\
\hline$r$, range $(\mathrm{m})$ & 130 & 190 & 570 & 700 \\
$d$, depth $(\mathrm{m})$ & 18 & 18 & 18 & 200 \\
\hline Input SNR (dB) & 45 & 37 & -2.5 & -0.7 \\
$\tau_{90}(\mathrm{~ms})$ & 9.6 & 1.3 & 20 & 21.4 \\
$\tau_{50}(\mathrm{~ms})$ & 0.04 & 0.4 & 1.4 & 3.6 \\
$f_{90}(\mathrm{~Hz})$ & 23.7 & 19.6 & 29.8 & 32.7 \\
$f_{50}(\mathrm{~Hz})$ & 2.1 & 7.5 & 10.3 & 10.3 \\
$\theta_{90}($ degrees $)$ & 36.6 & 23.9 & 150.5 & 154.7 \\
$\theta_{50}($ degrees $)$ & 9.8 & 7 & 40.7 & 45 \\
\hline \hline
\end{tabular}

$\mathrm{C}$ the direct arrival is hidden in a diffuse arrival of scattered paths. Channel D is the only deepwater channel, here there is a direct arrival followed by only a nearby surface reflection arriving $1 \mathrm{~ms}$ later. The reason for this is the high attenuation at $250 \mathrm{kHz}$, which strongly attenuates the sound traveling over longer distances. Channels D and C are low input signal-to-noise ratio (SNR) channels.

A summary of the conditions for the various experiments can be seen in Table I. The range is the horizontal distance from the transmitter to the receiver, the input SNR is defined as the ratio between background noise power measured before the signal and the signal power, averaged over the array. The delay $\left(\tau_{90}\right.$ and $\left.\tau_{50}\right)$, Doppler $\left(f_{90}\right.$ and $\left.f_{50}\right)$ and angular spread $\left(\theta_{90}\right.$ and $\left.\theta_{50}\right)$ are defined as the shortest intervals that respectively contain $90 \%$ and $50 \%$ of the received energy.

\section{RESULT AND DISCUSSION}

We will in this section present our result comparing the initial convergence and tracking capabilities for the RLS and the LMS algorithm.

\section{A. Comparison of tracking capabilities}

Fig. 4 shows the average stead state receiver output MSE for channel A-D, as function of the number of hydrophones after removing initial training and the minimum mean-square error 


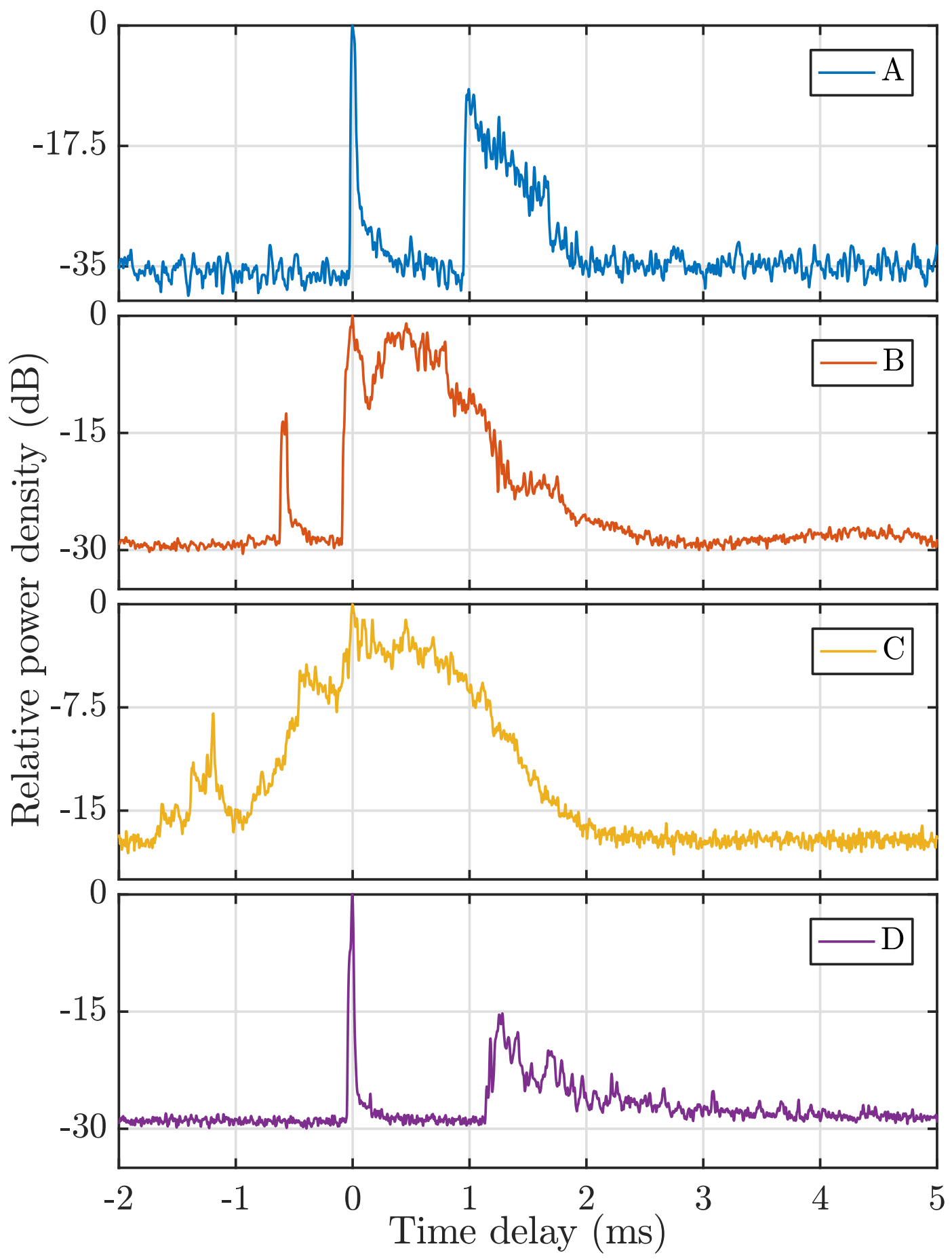

Fig. 2. Normalized power delay profile for channel A-D. 


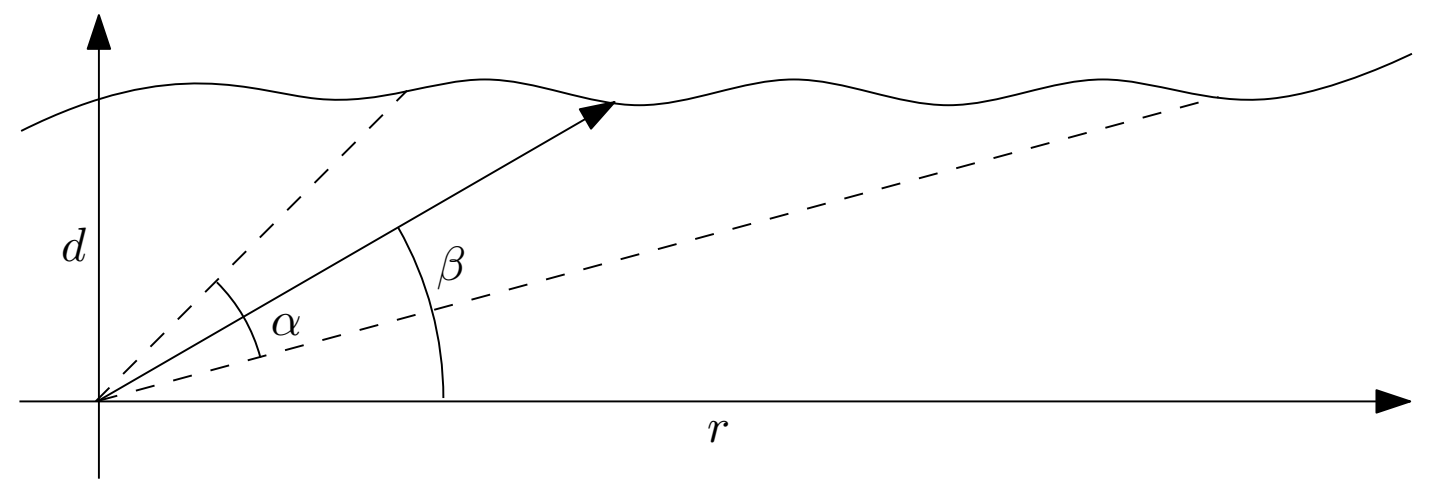

Fig. 3. Sketch of system setup, $r$ the horizontal range, $d$ the depth, $\alpha$ is the transducer opening angle, and $\beta$ the angle between the transducer center line and seabed.

equalizer bias. The array was populated within a fixed aperture. This gives a constant spatial resolution, in addition to the array gain but with the drawback of grating lobes potentially resulting in spatial under-sampling for the cases with few active hydrophones. The reason for populating a fixed aperture is to have as low spatial correlation as possible among the active hydrophones.

For all four channels the RLS equalizer has the best result, however the difference is only significant in channels A and B, and only when the number of active hydrophones are small. For channels C-D the difference is negligible, these channels are low SNR channels resulting in a failure of both the LMS and RLS algorithm to converge for few active hydrophones. For all channels, A-D, the reduction in MSE is fastest for the first number of added hydrophones. Channel B flattens out whereas the remaining channels have a steady reduction in MSE, although in the case of channel B the decrease is highly marginally. The reason for this is the spatial correlation, which is lowest for the first number of added hydrophones. In channel B no new information is added above 10 added hydrophones and it is also interesting to note that here the MSE increases slightly using RLS. For channel C and D that is low input SNR channels, a part of the continued reduction in MSE can be explained by array gain, this is however not the case for A which is a high input SNR channel suggesting that received signals are at-least partially uncorrelated within a few $\mathrm{cm}$ range. The reason for low spatial correlation in channel A can be explained via the energy rich and uncorrelated surface reflection arriving $1 \mathrm{~ms}$ after the direct arrival, Fig. 2.

The results presented in this section is as previously predicted, the difference in tracking 


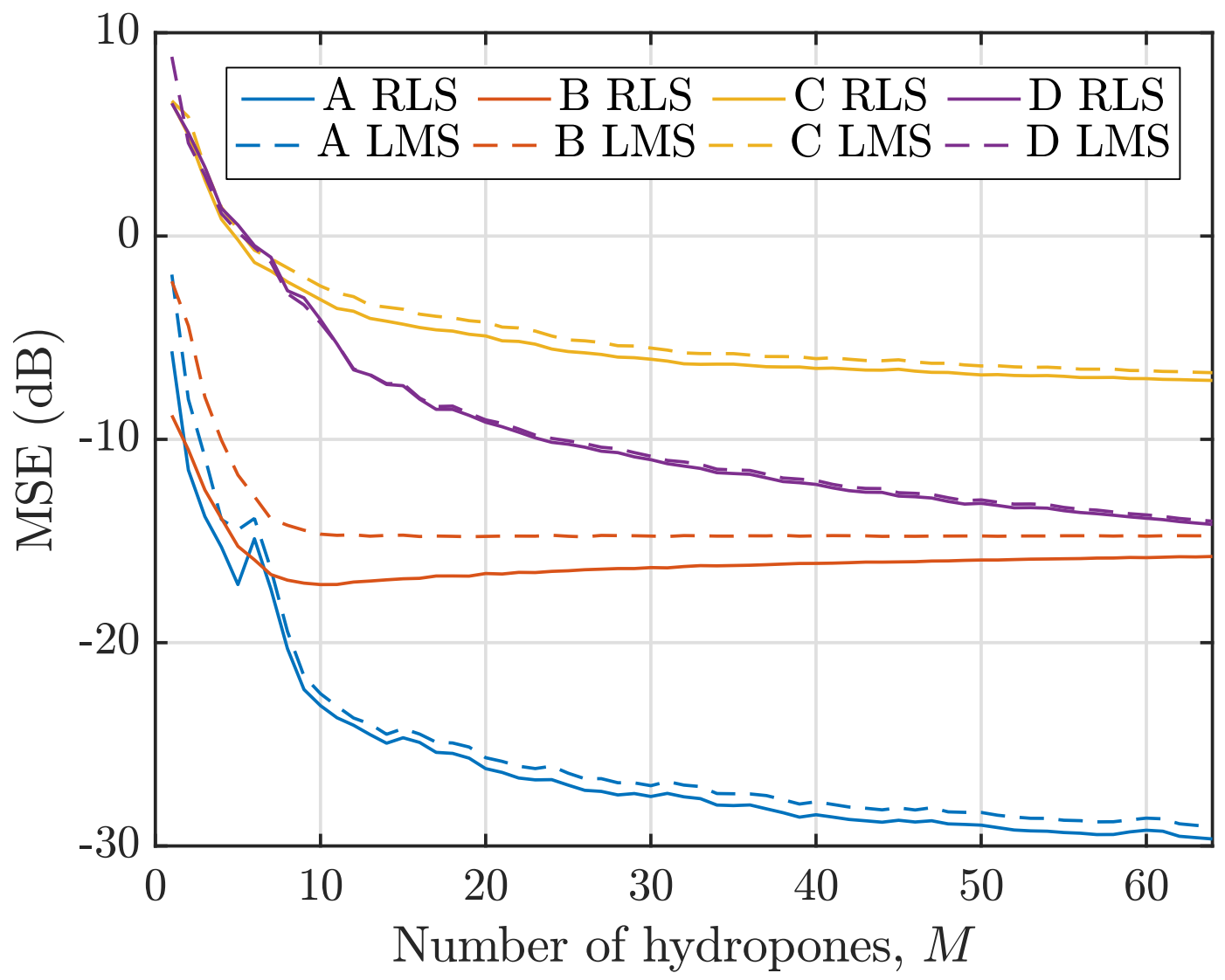

Fig. 4. Comparison of MSE using RLS and LMS for updating the receivers adaptive filter coefficients.

capability between RLS and LMS are reduced with increasing number of active hydrophones, to a level where they perform almost identical. Suggesting that for large arrays using adaptive equalizers LMS is a more suitable choice, both in high and low input SNR channels.

\section{B. Comparison of convergence}

A comparison for the initial convergence of channel A-D, can be seen in Fig. 6, the MSE has been filtered with a 100 tap boxcar filter in order to give a more readable appearance. We will here define convergence time as the time it takes to reach the mean value of the steady state MSE. Doing so the convergence rate has been plotted in Fig. 5, showing that the convergence rate is almost the same for both the RLS and LMS algorithm when the number of hydrophones increases. It is also interesting to note that the convergence rate in channel A, B and D increases with number of added hydrophones. 


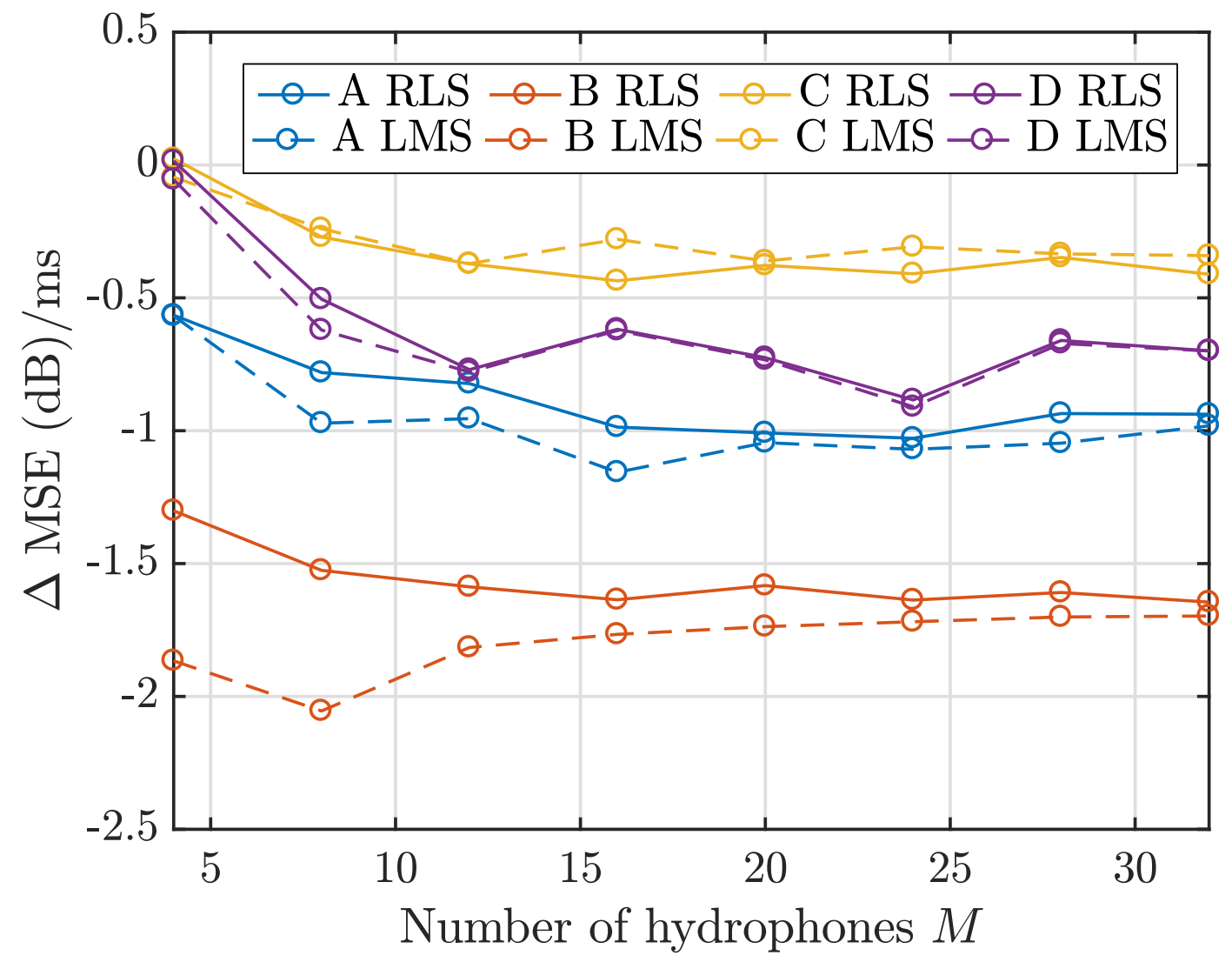

Fig. 5. Initial convergence rate comparison for RLS and LMS.

\section{CONCLuSiON}

We have in this paper compared the tracking performance and convergence rate of a multichannel equalizer using either the RLS or LMS algorithm to update its filter taps, for increasing number of contributing hydrophones in a line array. Evaluation of four different very high frequency acoustic channels suggests that when the number of contributing hydrophones is large, decisions feedback equalizers using less computationally demanding algorithm such as LMS perform similar to computationally heavy RLS algorithms. It does not seem that RLS offers neither a significant advantage over LMS when it comes to tracking channel variations, nor convergence rate, in multi-hydrophone equalizing systems.

\section{REFERENCES}

[1] P. A. van Walree, "Propagation and Scattering Effects in Underwater Acoustic Communication Channels," IEEE Journal of Oceanic Engineering, vol. 38, no. 4, pp. 614-631, Oct 2013. 


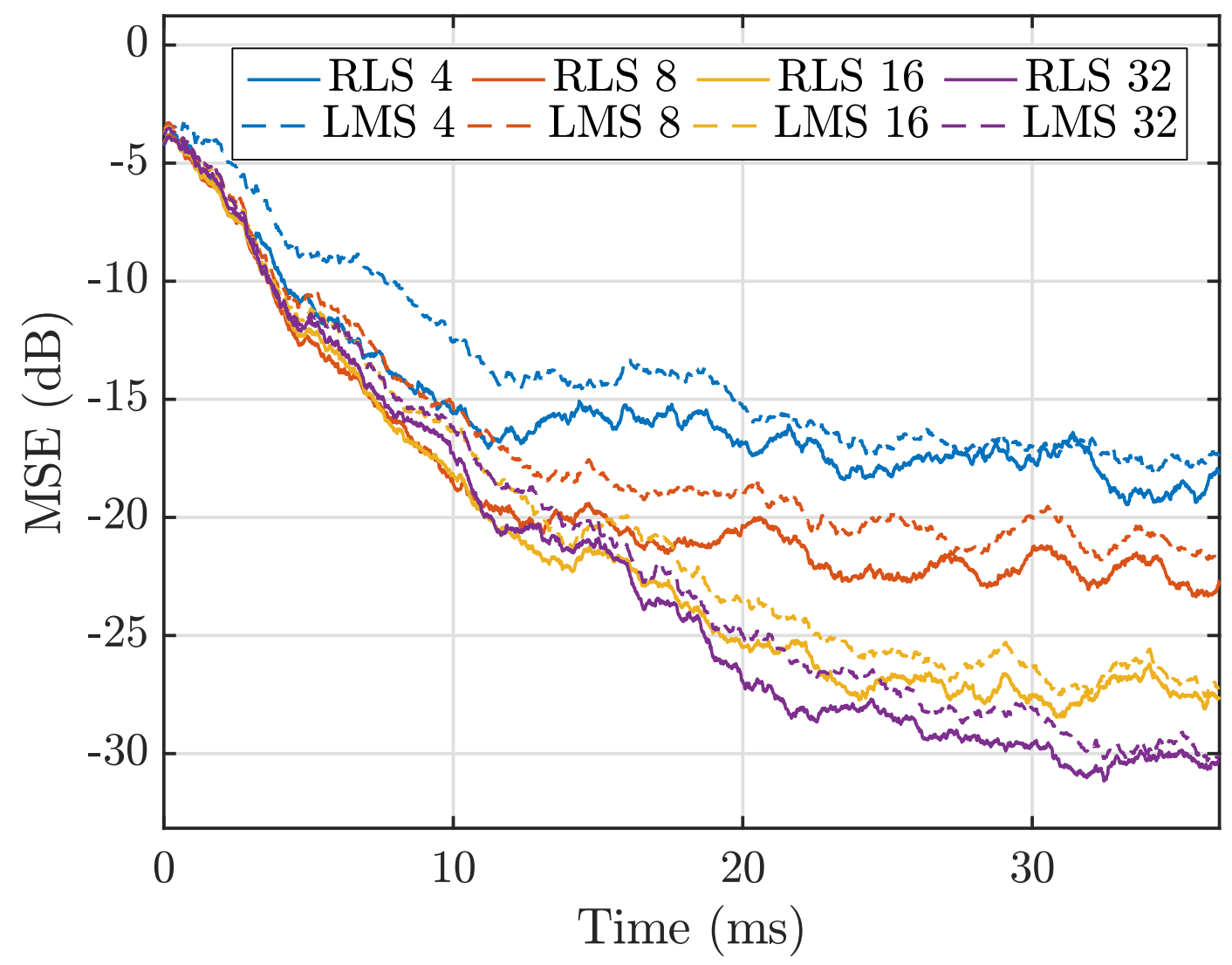

(a)

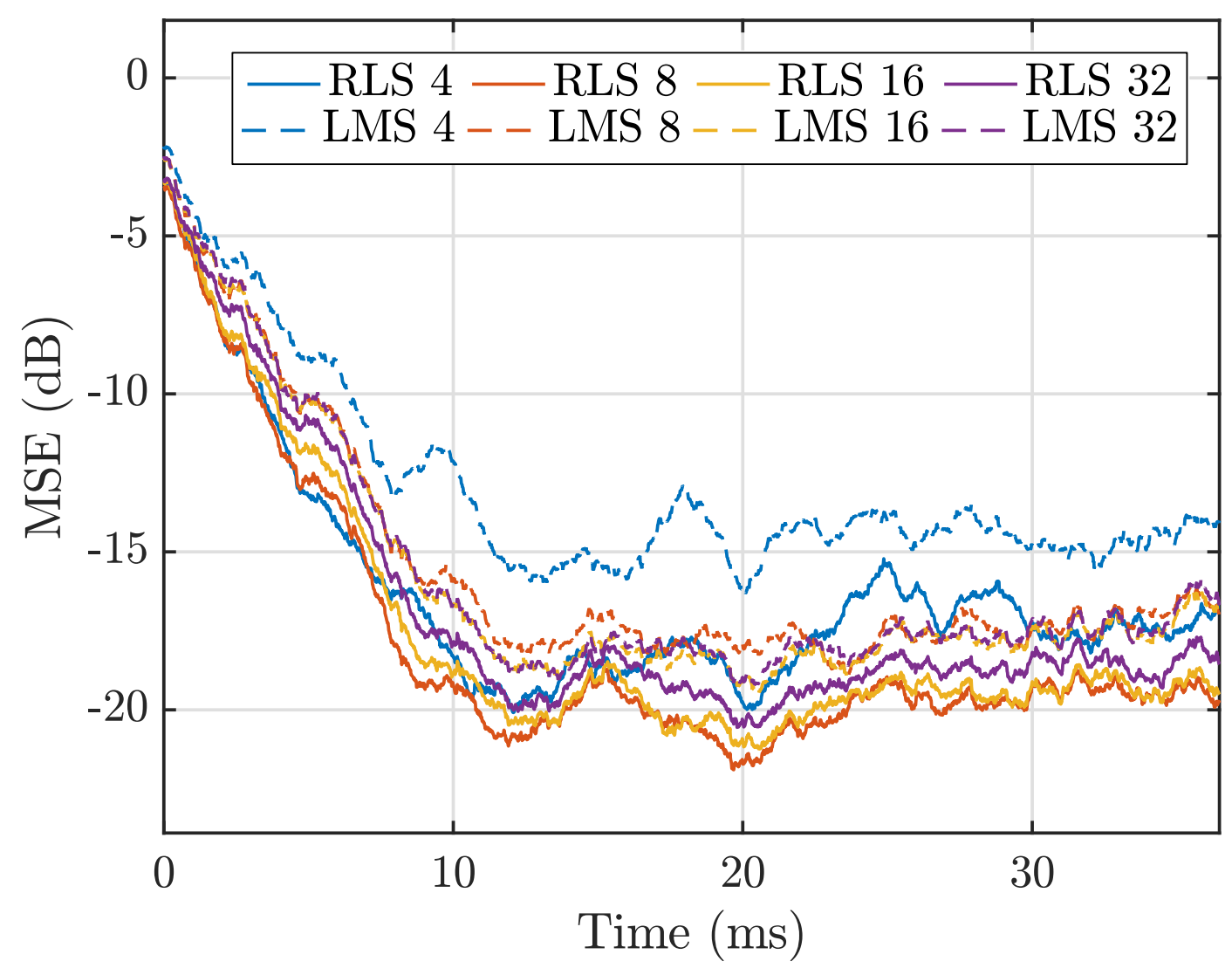

(b) 
[2] M. Stojanovic, J. A. Catipovic, and J. G. Proakis, "Phase-coherent digital communications for underwater acoustic channels," IEEE Journal of Oceanic Engineering, vol. 19, no. 1, pp. 100-111, Jan 1994.

[3] T. C. Yang, “A Study of Spatial Processing Gain in Underwater Acoustic Communications," IEEE Journal of Oceanic Engineering, vol. 32, no. 3, pp. 689-709, July 2007.

[4] M. Pajovic and J. C. Preisig, "Performance Analysis and Optimal Design of Multichannel Equalizer for Underwater Acoustic Communications," IEEE Journal of Oceanic Engineering, vol. 40, no. 4, pp. 759-774, Oct 2015.

[5] J. W. Choi, R. J. Drost, A. C. Singer, and J. Preisig, "Iterative Multi-Channel Equalization and Decoding for High Frequency Underwater Acoustic Communications," in 2008 5th IEEE Sensor Array and Multichannel Signal Processing Workshop, July 2008, pp. 127-130.

[6] J. Preisig, "Challenges and analysis of adaptive multichannel equalization for large-N arrays," in 2015 49th Asilomar Conference on Signals, Systems and Computers, Nov 2015, pp. 239-243.

[7] M. Stojanovic, J. A. Catipovic, and J. G. Proakis, "Reduced complexity spatial and temporal processing of underwater acoustic communication signals," The Journal of the Acoustical Society of America, vol. 98, no. 2, pp. 961-972, 1995.

[8] S. Haykin, Adaptive Filter Theory, 1st ed. New Jersey: Prentice-Hall, 1986.

[9] E. Eleftheriou and D. Falconer, "Tracking properties and steady-state performance of RLS adaptive filter algorithms," IEEE Transactions on Acoustics, Speech, and Signal Processing, vol. 34, no. 5, pp. 1097-1110, October 1986.

[10] J. Rudander, T. Husøy, P. Orten, and P. van Walree, "Shallow-water channel sounding for high speed acoustic communication," in OCEANS 2017 - Aberdeen, June 2017, pp. 1-8. 